\title{
Mechanism of Radiation Coupling to Plasma Wave Field Effect Transistor Sub-THz Detectors
}

\author{
M. Sakowicz, J. Łusakowski, K. Karpierz, M. Grynberg \\ Institute of Experimental Physics, University of Warsaw \\ Hoża 69, 00-681 Warsaw, Poland
}

W. GWAREK

Institute of Radioelectronics, Warsaw University of Technology

Nowowiejska 15/19, 00-665 Warsaw, Poland

W. KNAP* AND S. BOUbANGA

GES-CNRS URM 5650, Université Montpellier 2

Pl. Eugène Bataillon, 34950 Montpellier, France

\begin{abstract}
Detection of $100 \mathrm{GHz}$ and $285 \mathrm{GHz}$ electromagnetic radiation by GaAs/AlGaAs field effect transistors with the gate length of $150 \mathrm{~nm}$ was investigated at $300 \mathrm{~K}$ as a function of the angle $\alpha$ between the direction of linear polarization of the radiation and the symmetry axis of the field effect transistors. The angular dependence of the detected signal was found to be $A \cos ^{2}\left(\alpha-\alpha_{0}\right)+C$. A response of the transistor chip (including bonding wires and the substrate) to the radiation was numerically simulated. Calculations confirmed experimentally observed dependences and allowed to investigate the role of bonding wires and contact pads in coupling of the radiation to the transistor channel.
\end{abstract}

PACS numbers: 84.40.Ba, 52.40.Fd, 85.30.Tv, 07.57.Kp

\section{Introduction}

Field effect transistors (FETs) are known to be detectors of electromagnetic (EM) radiation of $\mathrm{THz}$ and sub-THz frequency. Under the illumination with EM wave, a constant voltage is created between the drain and the source. The physics of this photovoltaic effect is described by a model of the instability of the electron

*also Tohoku University RIEC Ultra-broadband Signal Processing, 2-1-1 Katahira, Aoba-ku 980-8577, Japan 
plasma in the channel of the transistor [1-4]. The photovoltage created with the radiation can be considered as resulting from the rectification of high frequency currents, induced by the radiation, in a nonlinear and asymmetric transistor channel. An important point is that the photovoltaic signal depends on the electron concentration in the channel, i.e., on the gate voltage, $U_{\mathrm{GS}}$, which offers the possibility to use FETs as voltage-tunable detectors of THz and sub-THz radiation. Performance of such detectors was tested both at liquid helium $[4,5]$ and room temperature [3, 6-8] on all relevant FET structures: GaAs/AlGaAs, GaN/AlGaN, GaInAs/AlInAs and Si metal-oxide-semiconductor FETs (MOSFETs).

One of questions related to this phenomenon concerns the coupling of the EM wave with the transistor channel. It is of a particular importance for potential applications since a strong coupling of the incident radiation with the transistor is crucial for a high sensitivity of a device. Since the wavelength of radiation is much larger than dimensions of the transistor channel, an antenna is necessary to provide an efficient coupling. The theory of plasma instability in FETs [1-4] does not consider details of the coupling. It is just assumed that the radiation couples with the channel through antenna. Authors do not specify what elements of the transistor make the antenna, i.e. the geometry of a real device is not analyzed. A correspondence between the theory and experimental data can be additionally perturbed by the fact that in a real experiment the transistor is bonded to a multipin support with thin wires. These elements become additional parts of an antenna which may influence the transistor response to the radiation. Lü et al. [9] indicated the importance of a proper orientation of the radiation polarization direction relatively to contact pads of transistors they used, but the problem was not analyzed in detail. Up to now, geometrical or directional aspects of coupling has not been experimentally investigated.

The purpose of the present work was to verify whether FET detection was sensitive to the polarization of the incident radiation. To this end, we carried out a detection experiment using a linearly polarized $100 \mathrm{GHz}$ and $285 \mathrm{GHz}$ radiation and commercially available GaAs/AlGaAs FETs. The detection signal (a constant source-drain voltage $S$ appearing as a response to the illumination) was measured both as a function of the gate voltage $U_{\mathrm{GS}}$ and as a function of the angle $\alpha$ between a symmetry axis of the transistor and the direction of polarization of the radiation. We show that the angular dependence of the detection signal forms a two-lobe pattern defined by $S(\alpha)=A \cos ^{2}\left(\alpha-\alpha_{0}\right)+C$, which clearly shows polarization effects in coupling of the incident radiation to the transistor chip, i.e. an enhanced sensitivity of a transistor for a particular direction of the radiation polarization, $\alpha_{0}$.

To understand experimental results for $100 \mathrm{GHz}$, we carried out numerical simulations of the response of a device to the incident radiation. A commercially available simulator, Quick-Wave 3D [10], was used to solve time-dependent Maxwell equations by the finite difference time domain (FDTD) method. Simula- 
tions comprised a realistic model of the device investigated (composed of the transistor on a semi-insulating GaAs substrate and bonding wires above the grounding plane) as well as the surrounding empty space. Results of the simulations agree with experimental data. They show, in particular, that the bonding wires and contact pads are parts of the antenna that couples the radiation to the channel but their relative role depends on the frequency of the radiation: above about $120 \mathrm{GHz}$, bonding wires become unimportant due to a large attenuation of high frequency currents in metal bonding wires. Experimental data is in agreement with this result.

\section{Experiment and results}

The transistors investigated were commercially available FUJITSU GaAs/AlGaAs high electron mobility FETs (HEMTs), FHX45X and FHX14X in the case of $100 \mathrm{GHz}$ and $285 \mathrm{GHz}$, respectively. The gate length of both FETs was shorter than $150 \mathrm{~nm}$ (as specified by the producer). Each transistor was processed on a separate chip with dimensions of about $0.5 \mathrm{~mm} \times 0.5 \mathrm{~mm}$. The transistor chip was glued to a commercial 14-pin dual-in-line ceramic support and contact pads were bonded to pins with $25 \mu \mathrm{m}$ thick aluminum wires (see Fig. 1). For both $100 \mathrm{GHz}$ and $285 \mathrm{GHz}$ sources emitted radiation was linearly polarized; the direction of polarization $(\alpha)$ was given by the geometry of the source output waveguide. The $100 \mathrm{GHz}$ source of the radiation was a Gunn diode operating in the cw mode. The diode was mounted in a support that enabled its rotation around a horizontal axis and we changed $\alpha$ by the diode rotation. The $285 \mathrm{GHz}$ source was a backward wave oscillator (BWO). We used a free standing wire polarizer to change the angle $\alpha$. A pyroelectric detector placed behind the transistor was used to monitor the power of the incident BWO radiation which was used to normalize the photovoltaic signal for each $\alpha$. The gate of the transistor was biased by a dc voltage source. A photovoltaic signal created between the drain and the source was measured by a standard lock-in technique with a mechanical chopper modulating the intensity of the incident radiation. The detection signal was measured as a function of the gate polarization $U_{\mathrm{GS}}$ for $\alpha$ between 0 and $360^{\circ}$ every $5^{\circ}$ or $10^{\circ}$.

White triangles in Fig. 1 show measured signal at $100 \mathrm{GHz}$ at $U_{\mathrm{GS}}=0$. The orientation of observed two-lobe pattern depends slightly on $U_{\mathrm{GS}}$; this dependence is discussed in detail in Ref. [11]. Similarly, results for $285 \mathrm{GHz}$ are shown in Fig. 2. Both data show clearly a strong anisotropy of the detection signal with sensitivity enhancement for one $\alpha$.

\section{Discussion}

Numerical simulations with a commercial simulator, Quick-Wave 3D (QW-3D) [10], were used to determine the distribution of the electric field in the chip and around the bonding wires in the case of $100 \mathrm{GHz}$ radiation. The 


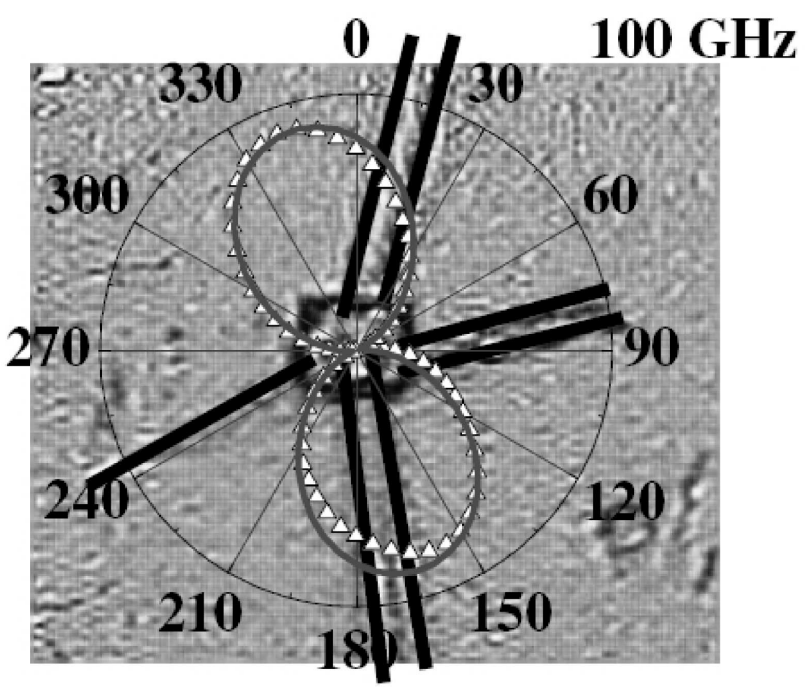

Fig. 1. A photograph of the chip (the rectangle in the center of the photograph) with the transistor investigated and bonding wires (thick black lines). The angular scale of the $\alpha$ angle is shown. Triangles: the polar plot of the measured signal at $U_{\mathrm{GS}}=0 \mathrm{~V}$; gray line: Quick-Wave 3D numerical simulations. Details of the configuration of the transistor electrodes are shown in Fig. 2.

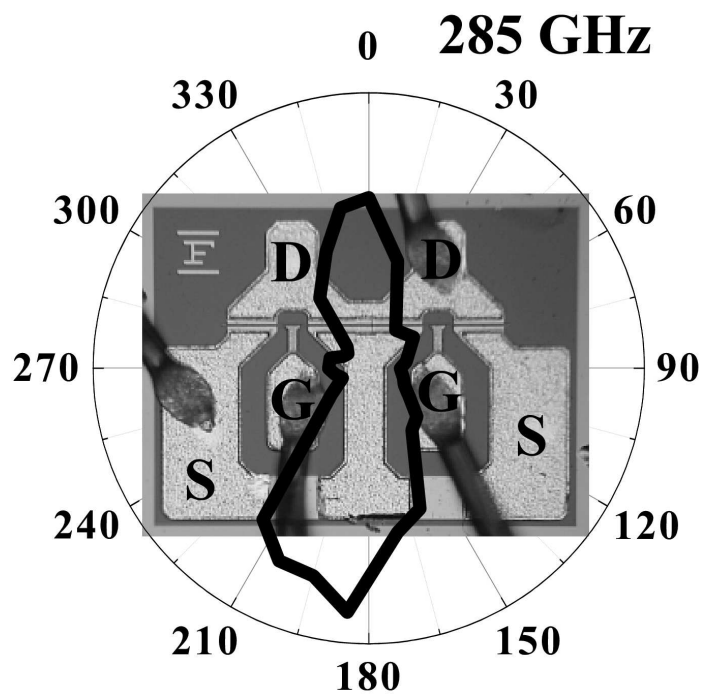

Fig. 2. A photograph of the transistor investigated with the gate (G), drain (D) and source (S) electrodes indicated. The angular scale of the $\alpha$ angle is shown. The black line shows the polar plot of the measured signal at $U_{\mathrm{GS}}=-0.3 \mathrm{~V}$. 
simulator solved time-dependent Maxwell equations by the finite difference time domain method. A simulation scenario was defined in which a realistic model of the transistor chip was attached to a ground plane with wires corresponding in length and direction to the actual bonding wires of the experiment. More details of the numerical procedure can be found in Ref. [11]. The gray line in Fig. 1 shows a very good agreement of the results of simulation with experimental data.

The main observations resulting from the simulations are the following. (1) The bonding wires make an important part of the antenna for $100 \mathrm{GHz}$ radiation frequency. It means that their orientation determines the angular sensitivity of the transistor. It is clear from Fig. 1 that the symmetry axis of the response does not coincide with the symmetry axis of the transistor, defined by $\alpha=0$. This is just caused by a "random" orientation of the bonding wires. (2) For frequencies higher than about $120 \mathrm{GHz}$, wires become not important. Therefore, in the case of $285 \mathrm{GHz}$ the angular response of the transistor is determined by its symmetry axis. (3) The dimensions of the channel and the contacts of the source and drain and the gate are big enough to cause different spatial field distributions in the volume of the chip (i.e., the substrate). At least two clearly distinct distributions (which may be called the substrate modes) were observed. The presence of the substrate modes additionally complicates the description of the effects of coupling of the radiation to the channel.

These results show that the dependence of the detection signal $S$ on the polarization of the incident radiation results from excitation of a few oscillators: bonding wires and substrate modes. In fact, at $100 \mathrm{GHz}$, the main elements responsible for the coupling are bonding wires which, by their nature, can be excited by a linearly polarized radiation. Thus, we should expect a response pattern resulting from a superposition of excitation of a few linearly polarized components with different polarization directions (defined by orientation of wires). For all these components oscillating in phase, the response would be like that of a single oscillator with a specific phase and orientation with respect to the phase and polarization vector of the incident wave. Then, excitation of a single (effective) oscillator would lead to a $S=A \cos ^{2}\left(\alpha-\alpha_{0}\right)$ dependence with $C=0$.

Experiments at $100 \mathrm{GHz}$ show, however, that the value of $C$ is comparable to the amplitude of the oscillatory part, $A$. This means that the excitation of the transistor chip can be described as resulting from two perpendicular phase-shifted oscillators which are equivalent to a single elliptically polarized oscillator. In such a case, neither $A$ nor $C$ can vanish. Simulations show that a phase shift between the oscillators can appear because the transistor structure resonates with two modes of a different phase shift.

In conclusion, we presented experimental and theoretical studies of polarization sensitive detection by high mobility FETs. Our results may help to design structures with a strong coupling of the radiation to the channel. It is also shown that FETs can be used to determine the polarization of sub-THz radiation. 


\section{Acknowledgments}

This work was supported by Polish 3T11B04528, 162/THz/2006/02 and European Union MTKD-CT-2005-029671 grants.

\section{References}

[1] M. Dyakonov, M.S. Shur, Phys. Rev. Lett. 71, 2465 (1993).

[2] M. Dyakonov, M.S. Shur, IEEE Trans. Electron Dev. 43, 380 (1996).

[3] D. Veksler, F. Teppe, A.P. Dmitriev, V.Yu. Kachorovskii, W. Knap, M.S. Shur, Phys. Rev. B 73, 125328 (2006).

[4] W. Knap, V. Kachorovskii, Y. Deng, S. Rumyantsev, J.-Q. Lü, R. Gaska, M.S. Shur, G. Simin, X. Hu, M. Asif Khan, C.A. Saylor, L.C. Brunel, J. Appl. Phys. 91, 9346 (2002).

[5] A. El Fatimy, F. Teppe, N. Dyakonova, W. Knap, D. Seliuta, G. Valušis, A. Shchepetov, Y. Roelens, S. Bollaert, A. Cappy, S. Rumyantsev, Appl. Phys. Lett. 89, 131926 (2006).

[6] F. Teppe, M. Orlov, A. El Fatimy, A. Tiberj, W. Knap, J. Torres, V. Gavrilenko, A. Shchepetov, Y. Roelens, S. Bollaert, Appl. Phys. Lett. 89, 222109 (2006).

[7] F. Teppe, W. Knap, D. Veksler, M.S. Shur, A.P. Dmitriev, V.Yu. Kachorovskii, S. Rumyantsev, Appl. Phys. Lett. 87, 052107 (2006).

[8] R. Tauk, F. Teppe, S. Boubanga, D. Coquillat, W. Knap, Y.M. Meziani, C. Gallon, F. Boeuf, T. Skotnicki, D.K. Maude, S. Rumyantsev, M.S. Shur, Appl. Phys. Lett. 89, 253511 (2006).

[9] J.-Q. Lü, M.S. Shur, J.L. Hesler, L. Sun, R. Weikle, IEEE Trans. Electron Dev. 19, 373 (1998).

[10] Quick-Wave 3D Electromagnetic Simulator, by QWED, Version 7.0, www.qwed.eu.

[11] M. Sakowicz, J. Łusakowski, K. Karpierz, M. Grynberg, W. Gwarek, J. Appl. Phys., in press. 